\title{
Variations of Boundary Surface in Chua's Circuit
}

\author{
Milan GUZAN \\ Dept. of Theoretical and Industrial Electrical Engineering, Technical University of Košice, \\ Park Komenského 3, 04001 Košice, Slovakia \\ milan.guzan@tuke.sk
}

\begin{abstract}
The paper compares the boundary surfaces using cross-sections in three projection planes, for the four changes of Chua's circuit parameters. It is known that due to changing the parameters, the Chua's circuit can be characterized not only by stable limit cycle, but also by one double scroll chaotic attractor, two single scroll chaotic attractors or other two stable limit cycles. Chua's circuit can even start working as a binary memory. It is not known yet, how changes in parameters, and consequently in attractors in the circuit, will affect the morphology of the boundary surface. The boundary surface separates the double scroll chaotic attractor from the stable limit cycle. In a variation of the parameters presented in this paper, the boundary surface will separate even single scroll chaotic attractors from each other. Dividing the state space into regions of attraction for different attractors, however, remains fundamentally the same.
\end{abstract}

\section{Keywords}

Chua's circuit, boundary surface, chaos

\section{Introduction}

Discovery of Chua's circuit initiated an explosion of publishing the results of his research. It is realistic to say that so far there have been more than a thousand papers published on Chua's circuit and its use [1]. Knowledge of Chua's circuit, or chaos, is also grouped into monographs, e.g. [2-5]. Continued activities related to chaos or to Chua's circuit prove topicality of this issue [6-9]. This paper composition was inspired by the reference [2], which enabled, in well-arranged and pedagogically illustrative way, to view entire gallery of chaotic attractors. Thus a natural question arose: How the variation of Chua's circuit parameters will affect the boundary surface (BS)?

What is BS? BS separates the individual attractors. The term BS is not only describing the separation of individual attractors in the circuit. In the plane, it is a separatrix, which divides the state plane into different large regions of attraction corresponding to particular attractors. The literature mentions not only the above terms (BS, separatrix, regions of attraction), but also the concepts rendering the same nature such as: basins of attraction or domains of attraction. Finding regions of attraction was already interesting before the onset of computer technology. Manually, using graphical methods, separatrix could be found in the circuit with a tunnel diode and thus find optimal parameters of the controlling impulse of bistable memory [10]. When designing and analyzing the elementary memories, it was, from engineering point of view, the main reason for studying BS. Knowledge of the separatrix could be used not only in the binary memory described by the set of two $(m=2)$ differential equations, but also in the m-dimensional state space $(m \geq 3)$ or at a later analysis (now using the computers) of multiple valued (MV) memory at $m=3$ [11-17]. In addition, knowledge of BS not only enables to design optimal parameters of controlling impulse of MV memory [16], but also to explain the failure of the memory - not only in the presence of stable limit cycles (SLCs), but also in their absence in case of virtual saddle point [14], [19-21]. This is another reason for interest in $\mathrm{BS}$ and its engineering usage.

Curve in the state plane (separatrix) at $m=2$ changes at $m \geq 3$ to the surface - BS and in the referred works [13-21] it was depicted through 2D cross-sections of BS. With the availability of computer technology, opportunities have arisen for the calculation of more trajectories depicting various regions of attraction not only in electrical circuits [11], [12], but also in other areas, where a set of differential equations could be used to describe the phenomenon analyzed [22]. Currently, knowledge of the basins of attraction is used e.g. in chaos circuit, robotics, engineering systems and neural networks [23-29].

Another reason for interest in study of BS lies in the exact explanations of activity of statistical sensors. Applying these sensors, enables to measure such small non-electric values, the size of which is, after the transformation to the electric variable, "drowned" in the noise of electric circuit. Finding equivalent voltage lies in "straightening" of deformed separatrix so that it began exactly at the beginning of the coordinate system. This will create same size regions of attraction for attractors "log. 0" or "log. 1". [30-35]. Since the author of work [10], studied BS for a long time, answer to the question raised by the author of Chua's circuit himself in [36]: What separates a chaotic attractor (CHA) from SLC?, was finally found. It was the BS that surrounded CHA and thus separated it from SLC. 
Thus BS in Chua's circuit received publicity for the first time in [37]. The meaning of BS in Chua's circuit rests not only in depicting its morphology in the state space. In case of using Chua's circuit to conceal the signal, it is impossible to comment on the maximum intensity of the concealed signal if we do not know the size of BS [36]. If the amplitude of the concealed signal exceeds BS, then modulation of the signal will not be unpredictable chaos, but very easily predictable periodic signal - SLC. This is another reason for interest of the author of this paper, and other researchers in [38], [39], [40] to study the morphology of $\mathrm{BS}$ in circuits generating chaos.

\section{Boundary Surface}

Knowledge of SC exceeds the sphere of the theory of nonlinear circuits, and the principles pronounced on BS can be used not only for the microchip, but in space too. In the previous section we demonstrated the use of BS. This section is devoted to defining BS.

Let's suppose, that the physical autonomous system is described by a set of ordinary differential equations in normal form

$$
\frac{\mathrm{d} \boldsymbol{x}}{\mathrm{d} t}=\boldsymbol{f}(\boldsymbol{x})
$$

where

$$
\boldsymbol{x}=\left[x_{1}, x_{2}, \ldots, x_{\mathrm{n}}\right]^{\mathrm{T}}
$$

and

$$
\boldsymbol{f}=\left[f_{1}, f_{2}, \ldots, f_{\mathrm{n}}\right]^{\mathrm{T}}
$$

whereby $\boldsymbol{f}(\mathbf{0})=\mathbf{0}$.

As it is a physical system, we assume that the system (1) satisfies the conditions of existence and uniqueness of a solution.

We refer to particular solutions by using a symbol ${ }^{\mathrm{i}} \boldsymbol{x}(t)$ whereby the upper index distinguishes particular solution leading to the corresponding attractor. To simplify further considerations, let's assume that the system (1) is characterized by two dynamic attractors $(\mathrm{A})-{ }^{\mathrm{i}} \boldsymbol{x}_{\mathrm{A}}(t)$ for $\mathrm{i}=1,2$. We will relate initial conditions (ICs) to $t=0$.

\section{Definition of $B S$}

Let's consider the following surface

$$
\varphi(\boldsymbol{x})=0
$$

that $\varphi\left[{ }^{1} \boldsymbol{x}_{\mathrm{A}}(t)\right]<0$ and $\varphi\left[{ }^{2} \boldsymbol{x}_{\mathrm{A}}(t)\right]>0$ for any $t \in T^{*}$, $T^{*}=(-\infty ;+\infty)$ and also particular solutions ${ }^{1} x(t),{ }^{2} x(t)$ for which applies the following

$$
\mathrm{d}\left[{ }^{1} \boldsymbol{x}(t),{ }^{1} \boldsymbol{x}_{\mathrm{A}}(t)\right] \rightarrow 0, \mathrm{~d}\left[{ }^{2} \boldsymbol{x}(t),{ }^{2} \boldsymbol{x}_{\mathrm{A}}(t)\right] \rightarrow 0
$$

for $t \rightarrow+\infty$. Symbol d $\left[{ }^{\mathrm{i}} x(t),{ }^{\mathrm{i}} x_{\mathrm{A}}(t)\right] \rightarrow 0$ is used to indi- cate the distance between the point ${ }^{\mathrm{i}} \boldsymbol{x}(t)$ and attractor ${ }^{\mathrm{i}} \boldsymbol{x}_{\mathrm{A}}(t)$. If for all particular solutions ${ }^{1} \boldsymbol{x}(t),{ }^{2} \boldsymbol{x}(t)$ suiting the relations (5), applies that $\varphi\left[{ }^{1} \boldsymbol{x}(t)\right]<0, \varphi\left[{ }^{2} \boldsymbol{x}(t)\right]<0$ for any $t \in T^{*}$, then we will refer to it as to surface (4) boundary surface [12].

\section{Chua's Circuit}

Chua's circuit in Fig. 1a) is described by the system (6), where $g\left(u_{1}\right)$ is in the algebraic form (7) of nonlinear piece-wise resistor $N R$. $R_{\mathrm{L}}$ is resistance of inductance $L$, for which applies $R_{\mathrm{L}}=0 . I$ is control pulse. In this article we consider $I=0$.

$$
\begin{gathered}
C_{1}\left(\frac{d u_{1}}{d t}\right)=G\left(u_{2}-u_{1}\right)-g\left(u_{1}\right)-I \equiv Q_{1}, \\
C_{2}\left(\frac{d u_{2}}{d t}\right)=G\left(u_{1}-u_{2}\right)+i \quad \equiv Q_{2}, \\
L\left(\frac{d i}{d t}\right)=-u_{2}-R_{L} i \quad \\
g\left(u_{1}\right)=m_{2} u_{1}+\frac{1}{2}\left(m_{1}-m_{0}\right)\left(\left|u_{1}-B_{P}\right|-\left|u_{1}+B_{P}\right|\right)+ \\
+\frac{1}{2}\left(m_{2}-m_{1}\right)\left(\left|u_{1}-B_{0}\right|-\left|u_{1}+B_{0}\right|\right) .
\end{gathered}
$$

Fig. 1b) depicts the cross-section of plane surfaces defined by system (8) with parameter values (9a). Because of $R_{\mathrm{L}}=0$, the singularities $\mathrm{P}^{-}, 0, \mathrm{P}^{+}$in Fig. $1 \mathrm{~b}$ ) are located in the plane $u_{2}=0$. All three singularities are special because they are unstable. It was unprecedented for physical circuits until the discovery of Chua's circuit. Therefore, the authors of the work [39] called them Chua's

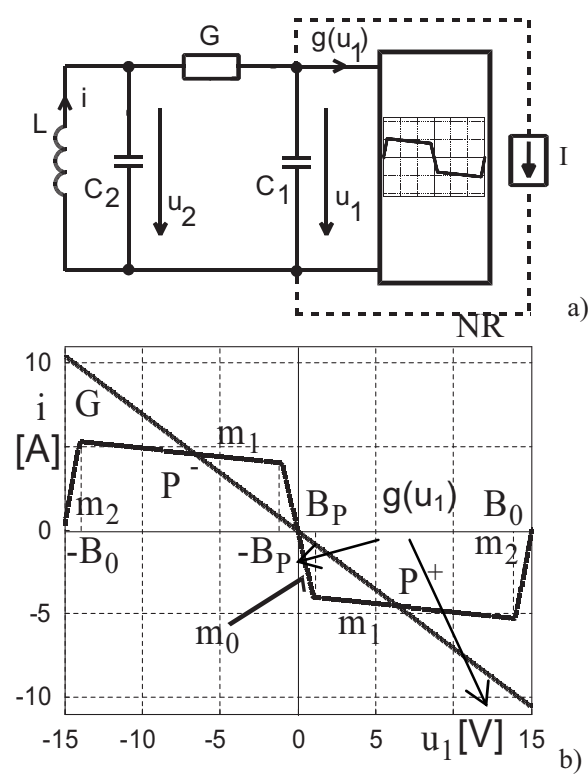

Fig. 1. a) Chua's circuit, b) I - V characteristics of conductance $G$ and $N R-g\left(u_{1}\right)$, with Chua's singularities $\mathrm{P}^{-}, 0, \mathrm{P}^{+}$. 

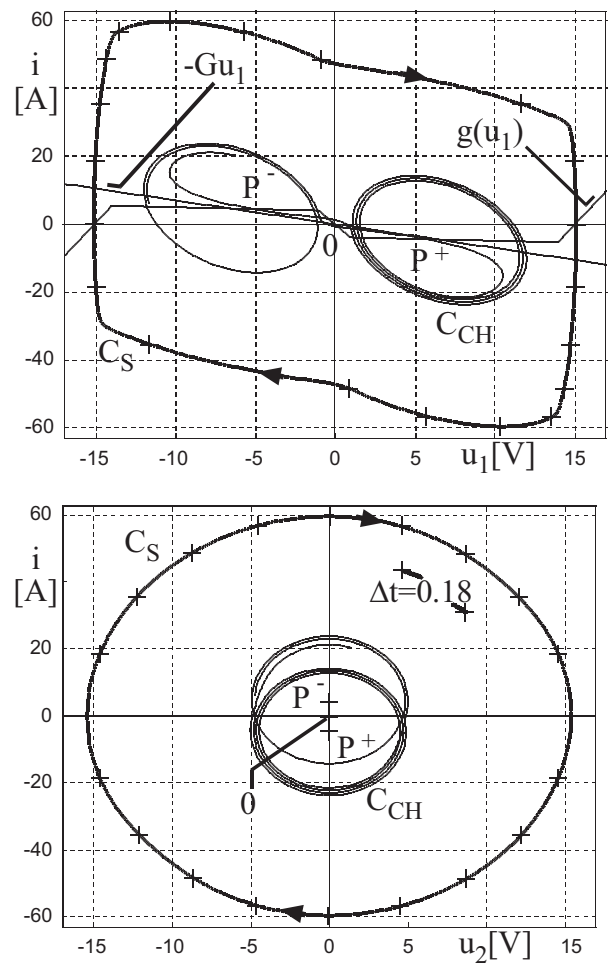

Fig. 2. The Monge projection of the chaotic attractor $\mathrm{C}_{\mathrm{CH}}$ and stable limit cycle $\mathrm{C}_{\mathrm{S}}$ for parameter values defined by (9a).

singularities. Thanks to three unstable singularities and parameters (4) the circuit is characterized by double scroll CHA (DCHA). The DCHA for parameters (9a) is illustrated in the Monge projection in Fig. 2 [39], where Cs is the SLC, $\mathrm{C}_{\mathrm{CH}}$ is DCHA and $\Delta \mathrm{t}$ is the time interval between the time label + . The arrow on SLC indicates the direction of representative point (RP) motion.

$$
\mathrm{Q}_{1}=0, \mathrm{Q}_{2}=0, \mathrm{Q}_{3}=0 \text {. }
$$

BS separating DCHA from SLC for parameters (9a) according to [39] is illustrated by cross-sections of BS in Fig. 3a). The gray area represents the region of attraction (RA) for DCHA and surrounding white RA for the SLC. It means that if RP is located in gray or white area of attraction, it will be attracted by either DCHA or SLC. Similar cross-sections of BS (as in Fig. 3a) are illustrated also in Fig. $3 b$ ) for the parameters (9b). Since the DCHA corresponding to parameters (9b) is smaller than DCHA illustrated in Fig. 3a), cross-sections of BS are also smaller in comparison to Fig. 3a). In principle, however, they remain the same. For $u_{1}<0$ cross-sections of BS are odd-symmetric. This fact is illustrated by Fig. 4 after reconstruction of BS in 3D [41]. Full 3D model consists of 300 calculated cross-sections of BS similar to those, illustrated in Fig. 4a).

$$
\begin{aligned}
& \frac{1}{C_{1}}=10, \quad \frac{1}{C_{2}}=0.5, \quad \frac{1}{L}=7, \quad G=0.7, \\
& m_{0}=-4, \quad m_{1}=-0.1, \quad m_{2}=5, \quad B_{P}=1 \text {, } \\
& B_{O}=14, \quad \rho=0, \quad I=0
\end{aligned}
$$

$$
\begin{aligned}
& \frac{1}{C_{1}}=9, \quad \frac{1}{C_{2}}=1, \quad \frac{1}{L}=7, \quad G=0.7, \\
& m_{0}=-0.8, \quad m_{1}=-0.5, \quad m_{2}=5, \quad B_{P}=1 \text {, } \\
& B_{O}=14, \quad \rho=0, \quad I=0 \text {. }
\end{aligned}
$$

\section{Variations of Boundary Surface}

In reference [2] in chapter The Physical Circuits there were total 195 CHA presented. For demonstration of changes in BS, first four CHA labelled PC1 - PC4 were chosen for this paper. Their parameters are shown in Tab. 1. From the time dependences presented in figures in [2] it was evident that PC1 and PC4 are double scroll CHA. The remaining CHA (PC2 and PC3) are single scroll CHA (SCHA). From Fig. 5a), however, is not obvious neither the size of attractors in the state space nor the size of BS.

\begin{tabular}{|c|c|c|c|c|}
\cline { 2 - 5 } \multicolumn{1}{c|}{} & PC1 & PC2 & PC3 & PC4 \\
\hline $\boldsymbol{C}_{\mathbf{1}}[\mathrm{nF}]$ & 0.104443 & 0.104443 & 0.104443 & 0.104443 \\
\hline $\boldsymbol{C}_{\mathbf{2}}[\mathrm{nF}]$ & 1 & 0.981 & 0.85 & 1 \\
\hline $\boldsymbol{G}[\mathbf{m S}]$ & 1.011 & 1 & 1 & 1 \\
\hline $\boldsymbol{R}_{\mathrm{L}}[\boldsymbol{\Omega}]$ & 0 & 0 & 0 & 0 \\
\hline $\boldsymbol{L}[\mathbf{m H}]$ & 0.0625 & 0.0625 & 0.0625 & 0.0625 \\
\hline $\boldsymbol{m}_{\mathbf{0}}[\mathrm{mS}]$ & -1.143 & -1.143 & -1.143 & -1.2 \\
\hline $\boldsymbol{m}_{\mathbf{1}}[\mathrm{mS}]$ & -0.714 & -0.714 & -0.714 & -0.714 \\
\hline
\end{tabular}

Tab. 1. Parameters for PC1-PC4 when considering $B_{\mathrm{p}}=1 \mathrm{~V}$.

In order to determine the number of attractors in the circuit and their size, the system (6) was solved using Runge-Kutta method. The program is written in $\mathrm{C}$ programing language. Because of simpler simulation, parameters in Tab. 1 were modified. According to [42] it applies, that for the purposes of simulation it is possible to use numerical parameters listed in Tab. 1 with the fact that $C_{1}$ and $C_{2}$ are in [F], $L$ is in [H], and conductivities $G, m_{0}, m_{1}$ in [S]. Tab. 1 is extended, in accordance with Fig. 2, to include parameters $B_{0}=14 \mathrm{~V}$ and $m_{2}=7 \mathrm{~S}$. Without these parameters, RP lying outside RA of CHA should be directed at infinity. Break point $B_{0}$ and conductance $m_{2}$ maintain physical nature of Chua's circuit model by attraction of RP to SLC, which is limited by a positive conductance $m_{2}$. New parameters $B_{0}$ and $m_{2}$, however, do not affect the existence and shape of the CHA, if the other parameters are from Tab. 1.

Simulation showed that circuits PC1-PC4 are characterized by:

- Two attractors - DCHA and SLC for cases PC1 and PC4,

- Three attractors - one SLC and two SCHA for cases PC2 and PC3.

This fact is illustrated by Fig. 5b) in projection into the $i, u_{1}$ plane. For aesthetic reasons, the figure does not depict neither whole I - V characteristics together with $B_{0}$ and $m_{2}$ nor SLC. The SLC would be huge compared to the 

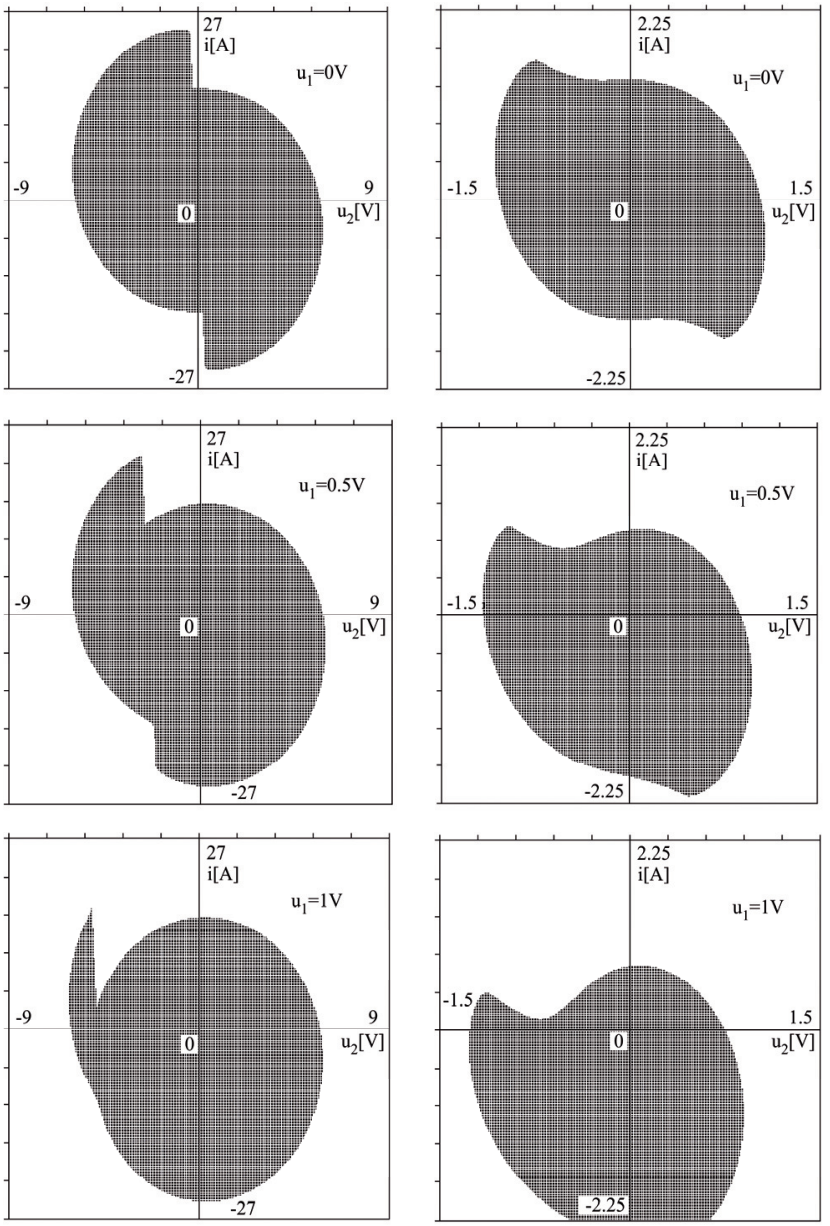

a)
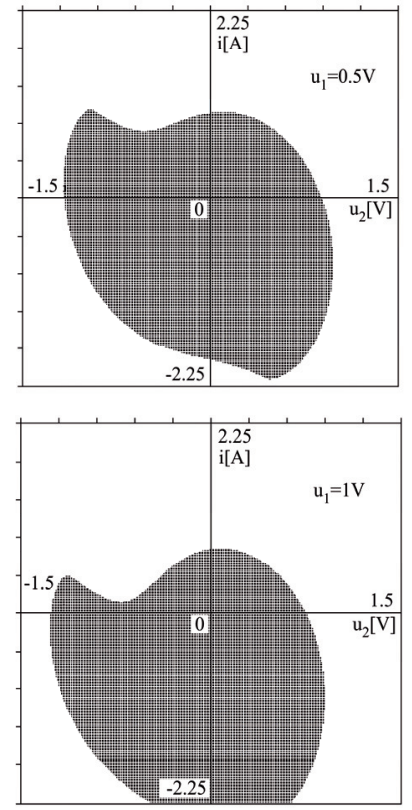

b)

Fig. 3. The cross-sections of the boundary surface with planes $u_{1}=0 \mathrm{~V} ; \quad 0.5 \mathrm{~V}$ and $1 \mathrm{~V}$ corresponding to [39]: a) parameters (9a), b) parameters $(9 b)$.

CHA, which are very small. ICs were chosen so that the image does not contain transient response, as seen for example from Fig. 5a) on PC1. ICs are part of the CHA. Fig. 5b) plots simultaneously both SCHA in PC2 and PC3, but for each SCHA different ICs were selected. Figure description for $\mathrm{PC} 3$ (axes, $\mathrm{P}^{-}, \mathrm{P}^{+}$etc.) is the same for the other cases of Fig. 5b). To distinguish DCHA from SCHA (figure PC2 looks like DCHA), DCHA are plotted in purple and two SCHA in green and red. The mentioned color distinction will be the color key for the next figures.

Looking at Fig. 5b), the question arises: What will be the morphology of BS due to variations in the parameters in Tab. 1? Grid technique [37] was used for calculation of cross-sections of BS through individual voltage and current levels. Simulation results are included in Fig. 6 - Fig. 8.

Figure 6 illustrates the cross-section of BS for PC1 to PC4 arranged in columns for $u_{2}=0, u_{1}=0$ and $i=0$. DCHA corresponds to parameters PC1 and PC4 and therefore, it is possible to note the similarity of cross-sections of BS for both cases. Yellow color represents (in all figures) RA for SLC, and purple color represents RA for DCHA illustrated in Fig. 5b) in purple color. The first picture in

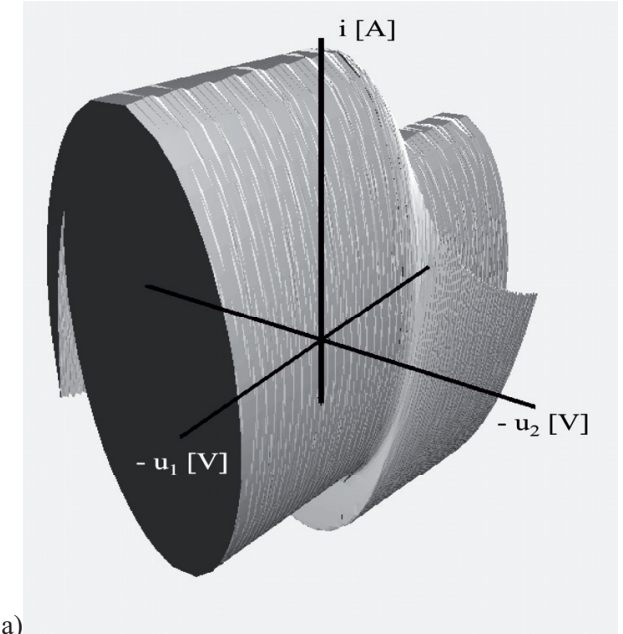

a)

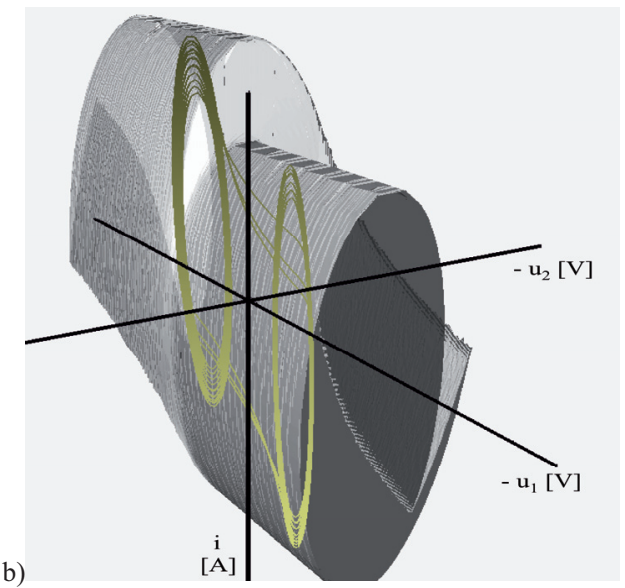

Fig. 4. Various view on reconstructed 3D BS of Chua's circuit for parameters (4a) [41]: a) opaque BS, b) transparent BS showing DCHA inside the BS.

each row in Fig. 6 also indicates axes description, size of the displayed plane and location of singularities for all next figures placed in rows. The rest of BS cross-sections in each line were calculated for the level indicated in the upper right corner of the first pictures in rows. A similar comment applies for Fig. 7 and Fig. 8.

Comparison of cross-sections of BS (for $u_{1}=0$ ) in Fig. 3 and especially in Fig. 3b) along with Fig. 6 for PC1 and PC4 showed that the RA for the cases of the DCHA are similar. Even for parameters $\mathrm{PC} 2$ and $\mathrm{PC} 3$ (cases of SCHA) in Fig. 6, the outer contour of RA neighboring on yellow RA is similar to violet RA. The difference is only in the "redistribution" of one RA for DCHA to two RA for SCHA. Figure 5b) for PC2 and PC3 shows color resolution of individual SCHA. RA for SCHA correspond to the same color region in Fig. 6, since the DCHA splits into two SCHA due to impact of parameters for PC2 and PC3 (Tab. 1). Purple inside of RA for DCHA is then divided into green and red RA for both SCHA and cross-section of $\mathrm{BS}$ commemorates the scroll. It is also interesting to note that the scroll BS is getting denser with going near the yellow RA corresponding to SLC attraction. 

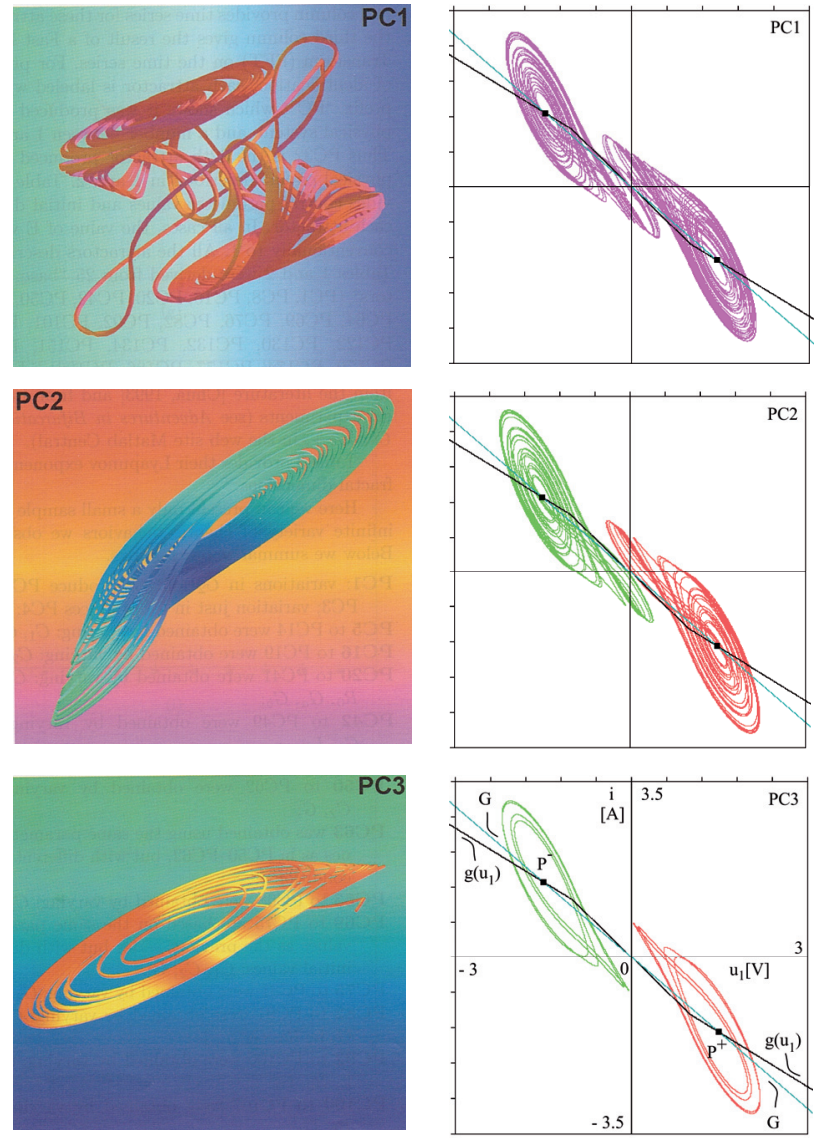

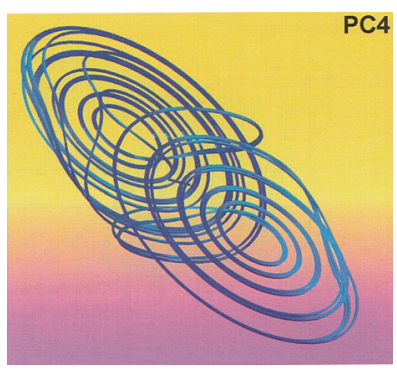

a)

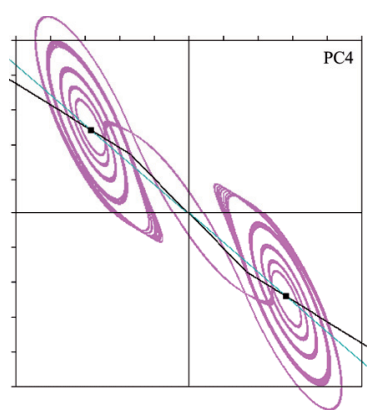

b)
Fig. 5. Chaotic attractors of Chua's circuit:

a) first four chaotic attractors chosen from [2],

b) for parameters of PC1 to PC4 listed in Tab. 1.

Size of the elements used in the simulation is specified according to $[41]$ in $[\mathrm{F}],[\mathrm{H}]$ and $[\mathrm{S}]$.

View of all cross-sections of BS illustrated in Fig. 6 allows not only imagining the state space dividing on RA corresponding to individual attractors, but also imagining the size of individual RA. Cross-section of BS for the PC1 is the smallest one and cross-sections of BS corresponding to PC3 and PC4 are about the same, although the DCHA PC4 in Fig. 5b) is the greatest of all CHA. Fig. 7 and Fig. 8 for $u_{2}=0.7 \mathrm{~V}$ and $u_{2}=1.3 \mathrm{~V}$ indicate that the BS in the direction of the axis $u_{2}$ will be narrow. The predominant yellow RA corresponding to SLC at $u_{2}=1.3 \mathrm{~V}$ (Fig. 8) proves it. In the $u_{1}$ axis direction the $\mathrm{BS}$ will be infinitely long with gradual vertical shift. The shift can be (similarly as in Fig. 4) seen in the Fig. 7 and Fig. 8 for cross-sections of BS in the plane $u_{1}=1.5 \mathrm{~V}$ and $u_{1}=3 \mathrm{~V}$. Because the BS for all four cases is a 3D object looking like pipe with "corn" on the side, further increase of current levels would lead again to one yellow area - RA for the SLC.

Comparing of Fig. 5b), where the maximum current $i_{\max }=3.5 \mathrm{~A}$ with cross-sections of BS in Fig. 8 for $i=3 \mathrm{~A}$, we find that CHA are not close to the BS (as shown in Fig. 4), but there is still a big gap between ${ }^{\mathrm{CHA}} i_{\max }$ and the BS. This indicates that the signal that should be encrypted can have greater amplitude than the ${ }^{\mathrm{CHA}} i_{\max }$ is. How wide the amplitude of signal exactly can be - it would require further analysis of the specific Chua's circuit. However, as shown in Fig. 8 (cross-sections of BS for $u_{1}=1.3 \mathrm{~V}$ ) the most limiting factor for the input signal will be voltage $u_{2}$.

In connection with the morphology of BS the question arises: Is it possible to find equations for the BS as functions of parameters of Chua's circuit? The answer can be summarized in the following points:

- If the BS for various parameters would have e.g. a form of a cylinder, or other object that can be described by the standard equations, the possibility of finding the equations is fair. Then there is no need to re-count BS cross-sections for each change of parameters.

- However, as seen e.g. in Fig. 7, Fig. 8 (for parameters PC1, PC4), but also in Fig. 3 and Fig. 4, although the BS has a cylindrical shape, the presence of a "corn" complicates this possibility.

- Finally, looking at Fig. 6-Fig. 8 (for parameters PC2 and PC3), where more and more fine changes of green and red colors toward the yellow region of attraction for SLC are visible, finding appropriate equations describing BS is impossible.

Therefore, it is necessary to re-calculate new cross-sections of BS at every change of circuit parameters, and only after the visualization of cross-sections, the possibility of describing BS by equations can be considered.

\section{Verification of Boundary Surface}

The accuracy of the calculation of cross-section of BS can be verified by calculation of several trajectories. For the calculation of the system (5) we selected a multipoint on the color boundary of BS. Calculated trajectory indicates if the RP is attracted to the corresponding attractor. As a demonstration of the calculation correctness, we selected two multi-points $\mathrm{A}$ and $\mathrm{B}$ for the case of PC3 in Fig. 6 in a plane $i, u_{2}$. In case of selection $\mathrm{A}$, it is a twopoint, its coordinates are in Tab. 2. The coordinates are also the ICs for the calculation of the system (5).

Figure 9 illustrates the Monge projection of trajectories calculated from IC1 and IC2 of two-point A. As it is evident from Fig. 9, the two trajectories are initially close to each other, but from a certain section they are already drifting apart. RP is attracted by green or red SCHA. The 

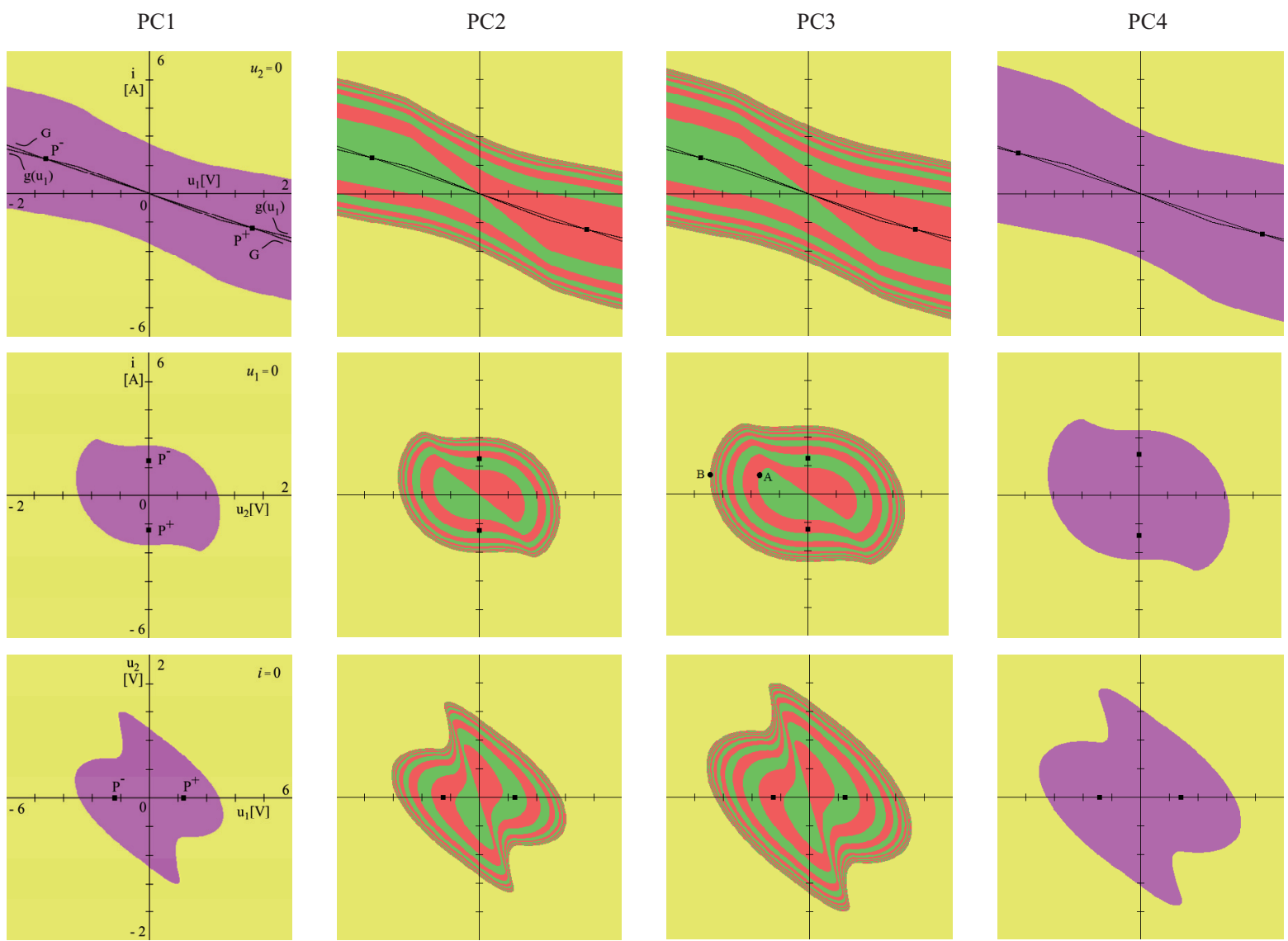

Fig. 6. Cross-sections of BS for PC1-PC4 through planes $u_{2}=0 ; u_{1}=0 ; i=0$.

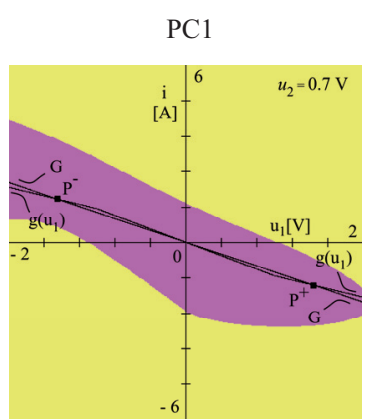

PC2

PC3
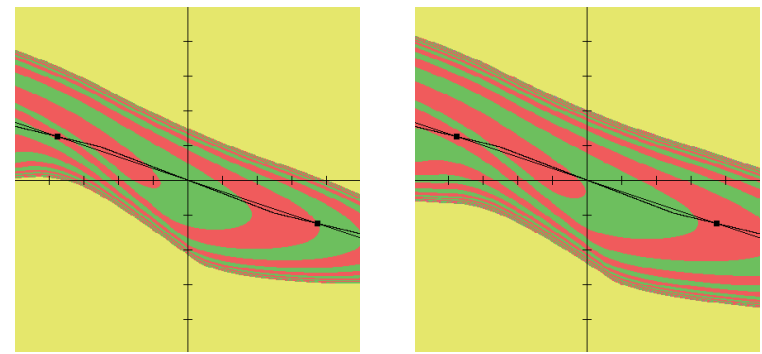

PC4
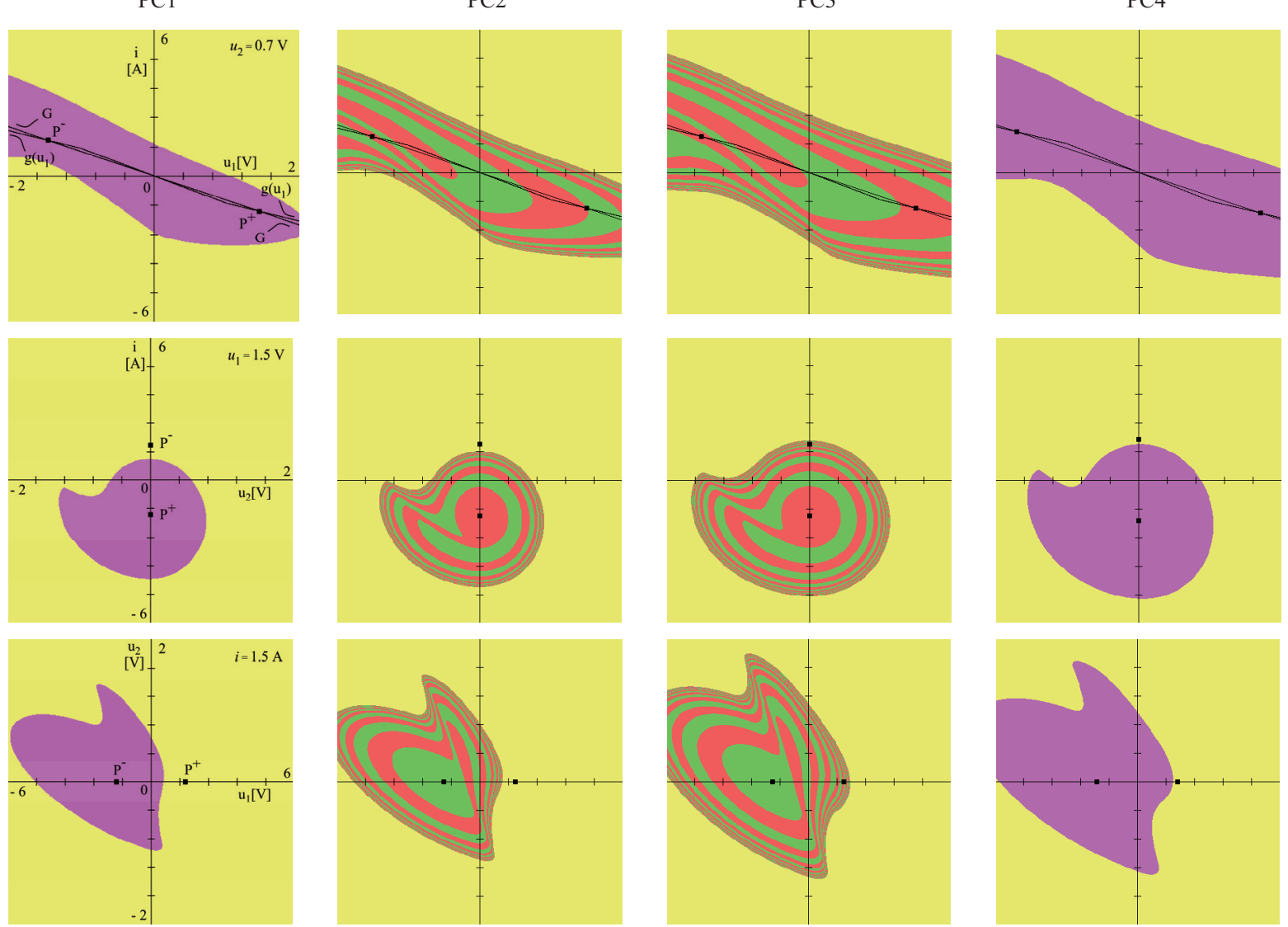

Fig. 7. Cross-sections of BS for PC1-PC4 through planes $u_{2}=0.7 \mathrm{~V} ; u_{1}=1.5 \mathrm{~V} ; i=1.5 \mathrm{~A}$.

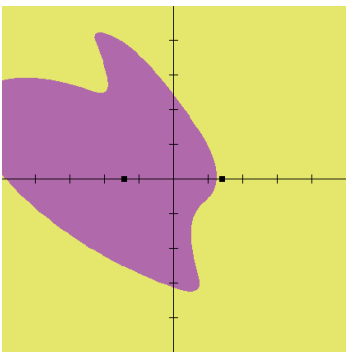



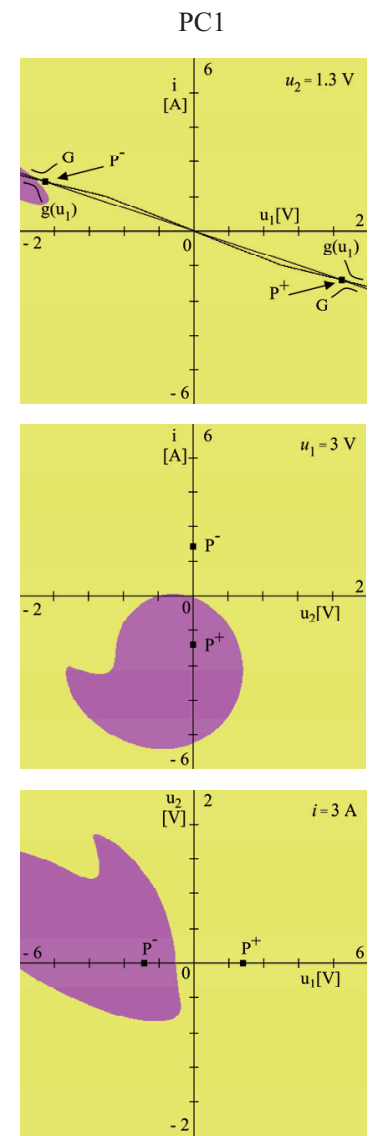

PC2
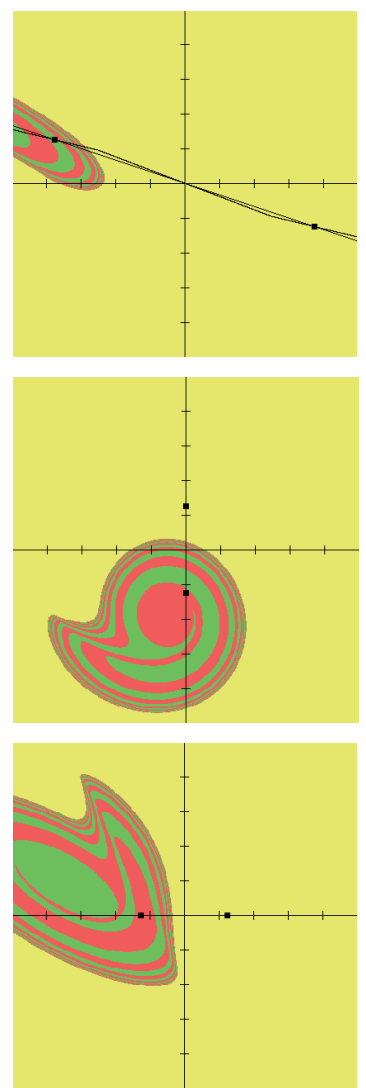

$\mathrm{PC} 3$
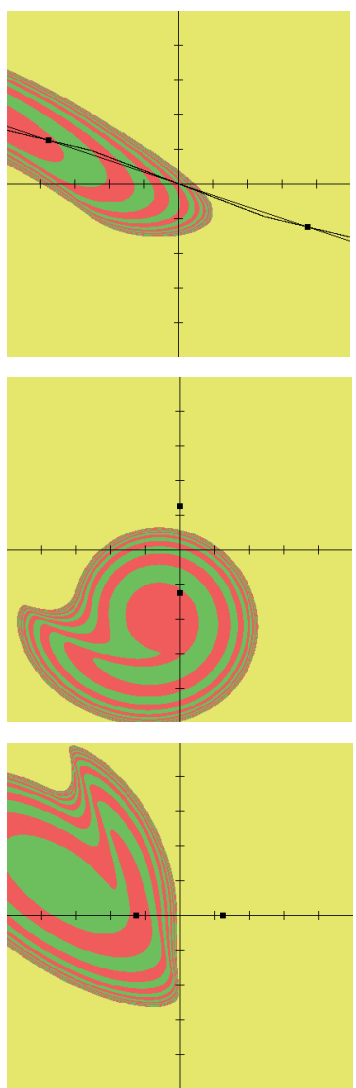

PC4
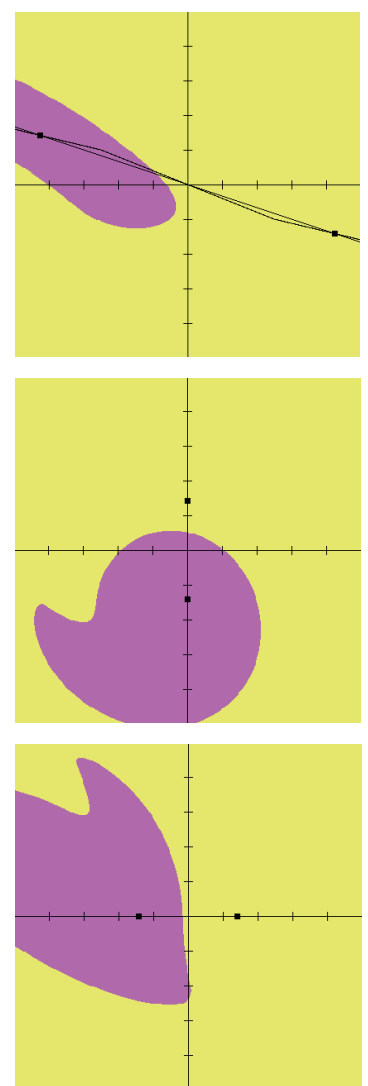

Fig. 8. Cross-sections of BS for PC1-PC4 through planes $u_{2}=1.3 \mathrm{~V} ; u_{1}=3 \mathrm{~V} ; i=3 \mathrm{~A}$.

arrows indicate the direction of RP motion. This picture and other pictures in Fig. 10-Fig. 12 graphically illustrate the validity of equation (5). IC1 and IC2 are located on opposite sides of the BS, and it is not possible for the RP alone without the external signal to exceed the BS and then being drawn by another attractor.

\begin{tabular}{|c|c|c|c|c|c|}
\cline { 3 - 6 } \multicolumn{2}{|c|}{} & $\boldsymbol{i}[\mathbf{A}]$ & $\boldsymbol{u}_{2}[\mathbf{V}]$ & $\boldsymbol{u}_{1}[\mathbf{V}]$ & Attractor \\
\hline \multirow{4}{*}{ A } & IC1 & 0.709086 & -0.690910 & 0 & red SCHA \\
\cline { 2 - 6 } & \multirow{2}{*}{$\mathbf{I C 2}$} & 0.709086 & -0.681819 & 0 & $\begin{array}{c}\text { green } \\
\text { SCHA }\end{array}$ \\
\hline
\end{tabular}

Tab. 2. Initial conditions corresponding to two-point A.

Figure 10 illustrates three trajectories calculated from three-point B. The coordinates of each IC are listed in Tab. 3. Initial conditions IC1 lead to SLC. For visual demonstration of the trajectory we replaced the yellow color corresponding to region of attractions for SLC (Fig. 6) by the blue color trajectory. SLC in Fig. 10 is compared to the green and red SCHA, so Fig. 11 illustrates the detail, which captures trajectories for the three ICs: IC1, IC2 and IC4. The figure clearly shows the thick "belt" of trajectories, which is related to BS.

From the beginning, all three trajectories are close together, then each of the RP is attracted by an attractor to different side of state space. Figure 12 illustrates now only two trajectories leading to green and red SCHA, similarly as shown in Fig. 9. Since the multipoint B is located on the outer boundary of regions of attraction SLC and SCHA, for three RPs it takes much longer time till they are attracted by attractors, than in case of Fig. 9 .

\begin{tabular}{|c|c|c|c|c|c|}
\cline { 2 - 6 } \multicolumn{2}{c|}{} & $\boldsymbol{i}[\mathbf{A}]$ & $\boldsymbol{u}_{\mathbf{2}}[\mathbf{V}]$ & $\boldsymbol{u}_{\mathbf{1}}[\mathbf{V}]$ & Attractor \\
\hline \multirow{4}{*}{$\mathbf{B}$} & IC1 & 0.709086 & -1.372728 & 0 & yellow - SLC \\
\cline { 2 - 6 } & IC2 & 0.709086 & -1.363637 & 0 & red SCHA \\
\cline { 2 - 6 } & IC3 & 0.709086 & -1.354546 & 0 & red SCHA \\
\cline { 2 - 6 } & IC4 & 0.709086 & -1.345455 & 0 & green SCHA \\
\hline
\end{tabular}

Tab. 3. Initial conditions corresponding to multipoint B.

Since the BS for PC2 and PC3 is significantly different from the $\mathrm{BS}$ for $\mathrm{PC} 1$ and $\mathrm{PC} 4$, the trajectories illustrated in Fig. 9-Fig. 12 illustrate the correctness of the BS calculation for all four cases. It is interesting that whether Chua's circuit is characterized by double scroll CHA (PC1, PC4) or by pair of single scrolls CHA (PC2, $\mathrm{PC} 3$ ), the $\mathrm{BS}$ is always characterized by the presence of "corn". The "corn" can be seen for example in Fig. $8\left(u_{1}=\right.$ $3 \mathrm{~V})$, but also for other parameters as (9a) and (9b) - see Fig. 3, Fig. 4. It will therefore be interesting to track the impact of multi-scroll CHA published in [2], [43], [44] on the presence of "corn" and on the morphology of BS. 


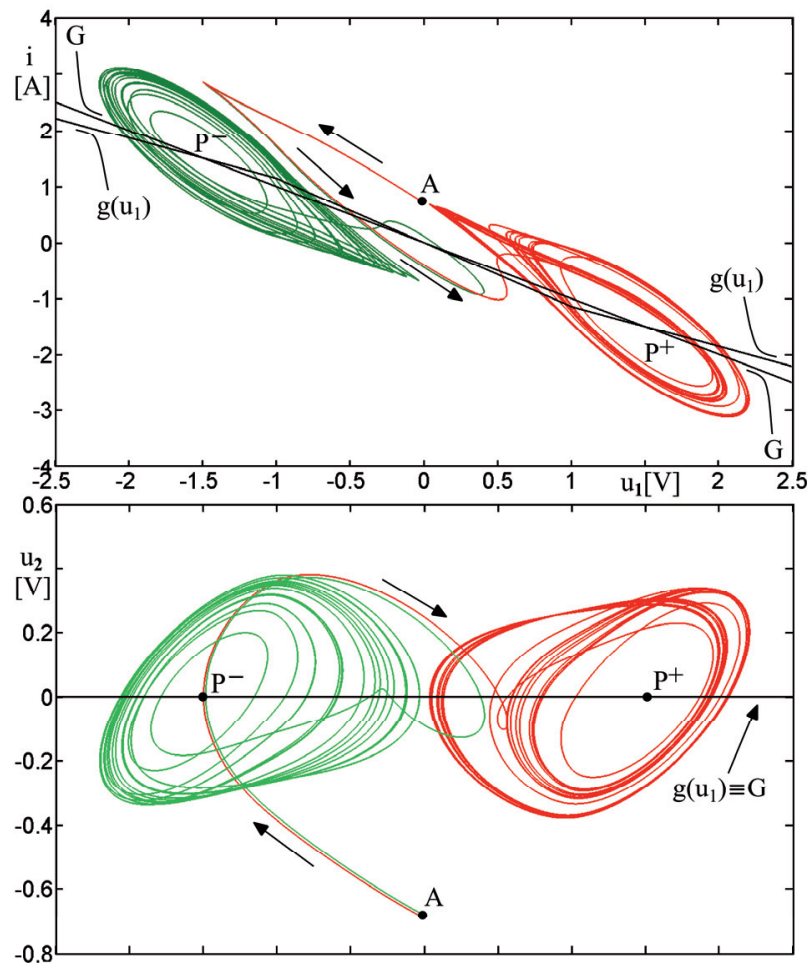

Fig. 9. Monge projection of two trajectories calculated from two-point A (Fig. 6, PC3, $u_{1}=0$ ) for ICs listed in Tab. 2.
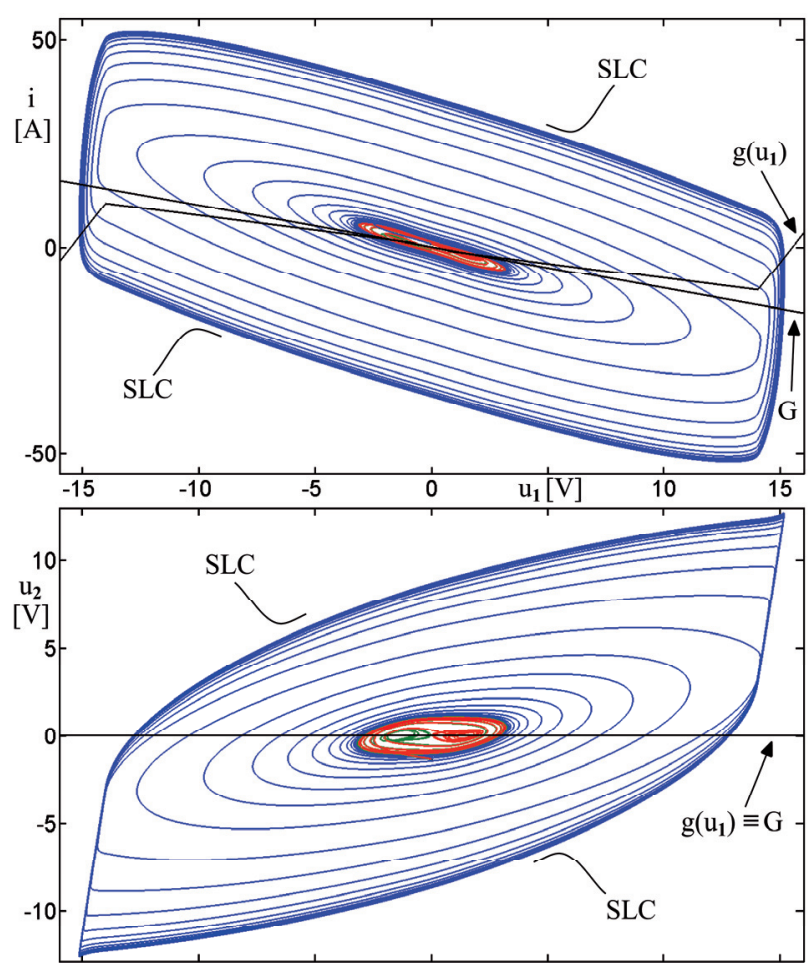

Fig. 10. Monge projection of three trajectories calculated from multipoint B (Fig. 6, PC3, $u_{1}=0$ ) for IC1, IC2 and IC4 listed in Tab. 3.
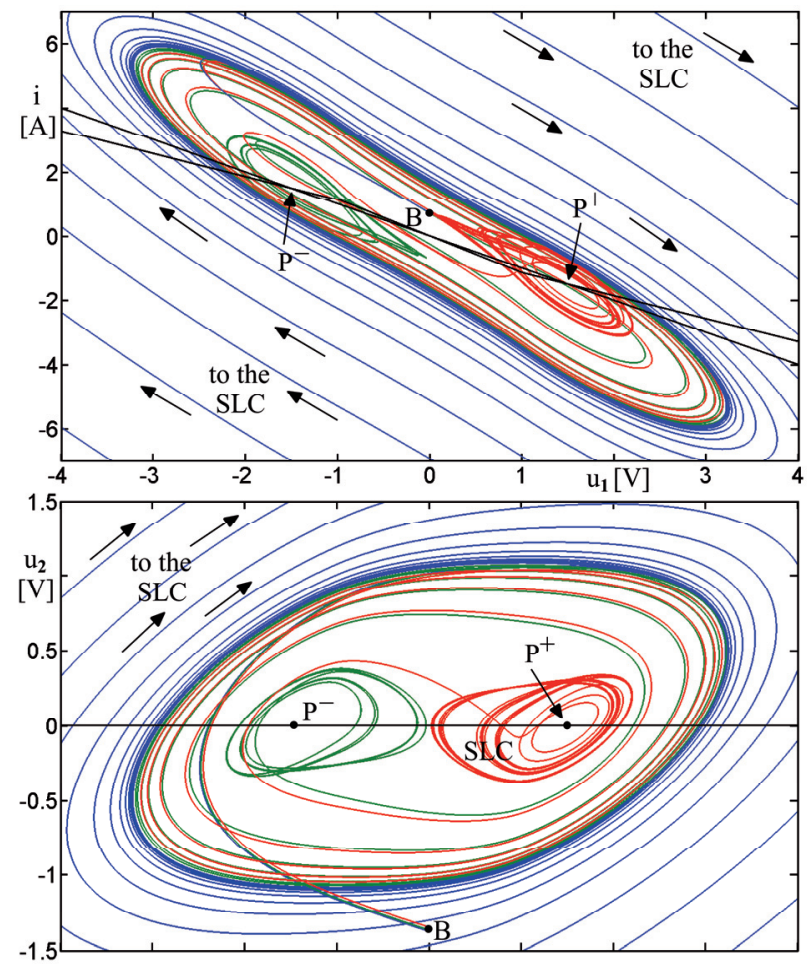

Fig. 11. Detail of trajectories in Fig. 10.
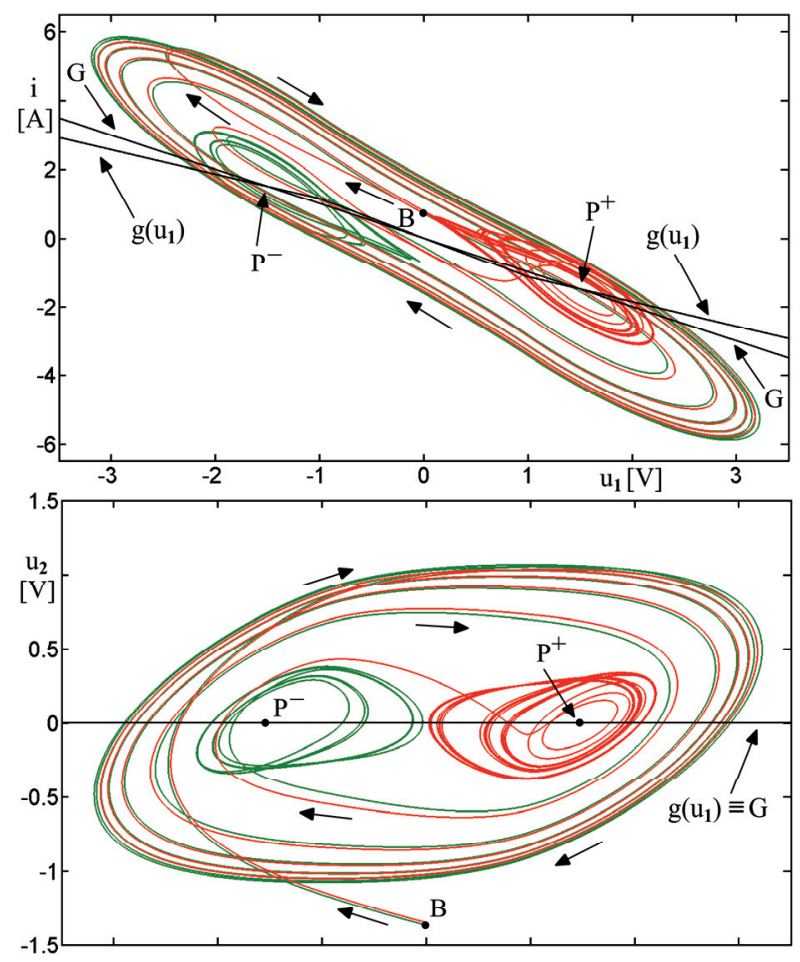

Fig. 12. Monge projection of two trajectories calculated from IC2 and IC4 (multipoint B) in Tab. 3. 


\section{Conclusion}

This paper has compared BS cross-sections for four variations of Chua's circuit parameters. It was found that the RA for CHA surrounded by a yellow RA for SLC are principally the same. It should be noted, however, that all four cases relate to $R_{\mathrm{L}}=0$. In reference [2] however also such cases are considered, when $R_{\mathrm{L}} \neq 0$. In chapter The Physical Circuits cases are presented, where $R_{\mathrm{L}}$ is in the range from $-0.1 \Omega$ to $12.1 \Omega$. It is now difficult to say how $R_{\mathrm{L}} \neq 0$ or multi-scroll CHA will affect the BS morphology for such cases. This will be the subject of further research of the author.

\section{Acknowledgments}

Research described in this paper was supported through project number GA15-22712S and the project: We support research activities in Slovakia. Project is co-financed from EU funds. This paper was developed within the Project "Centre of Excellence of the Integrated Research \& Exploitation the Advanced Materials and Technologies in the Automotive Electronics", ITMS 26220120055

\section{References}

[1] Chua's Circuit and Chua's Equation. [Online] Cited 2013-05-31. Available at: http://www.eecs.berkeley.edu/ chua/circuitrefs.html.

[2] BILOTTA, E., PANTANO, P. A. Gallery of Chua Attractors. World Scientific Series on Nonlinear Science Series A, 2008, vol. 61. ISBN: 978-981-279-062-0

[3] FORTUNA, L., et al. Chua's Circuit Implementations. World Scientific Series on Nonlinear Science Series A, 2009, vol. 65. ISBN: 978-981-283-924-4

[4] KILIÇ, R. A. Practical Guide for Studying Chua's Circuits. World Scientific Series on Nonlinear Science Series A, 2010, vol. 71, ISBN: 978-981-4291-13-2

[5] ADAMATZKY, A., CHEN, G. Chaos, CNN, Memristors and Beyond. World Scientific, 2013. ISBN: 978-981-4434-79-9

[6] MKAOUAR, H., BOUBAKER, O. On electronic design of the piecewise linear characteristic of the Chua's diode: Application to chaos synchronization. In Proceedings of the 16th IEEE Mediterranean Electrotechnical Conference (MELECON). 2012, p. 197-200. DOI: 10.1109/MELCON.2012.6196412

[7] FEKI, M., El GAMMOUDI, I. Chaos in Chua circuit with fractional order low pass filter. In Proceedings of the 8th International Multi-Conference on Systems, Signals and Devices (SSD). 2011, p. 1-4. DOI: 10.1109/SSD.2011.5986785

[8] XIAORAN LIN, SHANGBO ZHOU, HUA, LI. Chaos in complex-order Chua's system and its application to secure communication. In Proceedings of the Second International Conference on Communication Systems, Networks and Applications (ICCSNA). Hong Kong, 2010, p. 204-207. DOI: 10.1109/ICCSNA.2010.5588689

[9] GONG-BIN QIAN, QING-FENG JIANG, SHUI-SHENG QIU. A new image encryption scheme based on DES algorithm and
Chua's circuit. In Proceedings of the IEEE International Workshop on Imaging Systems and Techniques (IST). Shenzhen (China), 2009, p. 168-172. DOI: 10.1109/IST.2009.5071626

[10] ŠPÁNY, V. The analysis of the one-tunnel diode binary. Proceedings of the IEEE, 1967, vol. 55, no. 6, p. 1089-1090. DOI: 10.1109/PROC.1967.5737

[11] ŠPÁNY, V. Special surfaces and trajectories of the multidimensional state space. In Proceedings of Scientific Works of VŠT in Košice, part. 1. Košice (Slovakia), 1978, p. 123-152. (In Slovak)

[12] ŠPÁNY, V. Multistable systems and special surfaces of the mdimensional state space. Elektrotechnický časopis, 1982, vol. 33, no. 7 , p. 551-565. (In Slovak)

[13] ŠPÁNY, V., GALAJDA, P., GUZAN, M. Boundary surfaces of one-port memories. In Proceedings of Conference Tesla III Millennium. Belgrade (Yugoslavia), 1996, p. IV-131-137.

[14] GALAJDA, P., GUZAN, M., ŠPÁNY, V. The state space mystery with negative load. Radioengineering, 1999, vol. 8, no. 2, p. 2-7. ISSN 1210-2512.

[15] GUZAN, M. Boundary surface and stable manifold in sequential circuits. In Proceedings of 21th International Conference Radioelektronika 2011. Brno (Czech Republic), 2011, p. 219-222. DOI: 10.1109/RADIOELEK.2011.5936476

[16] GUZAN, M., SOBOTA, B. Boundary surface of multiple-valued memory in 3D. In Proceedings of the International Conference of Teachers of Electrical Engineering SEKEL. 2012, p. 75-80. (In Slovak)

[17] GUZAN, M. Boundary surface of 5-valued memory. Journal of Engineering, Hindawi Publishing Corporation, 2013, vol. 2013, p. 1-7. DOI: $10.1155 / 2013 / 626824$

[18] GALAJDA, P., GUZAN, M., ŠPÁNY, V. The control of a memory cell with the multiple stable states. In Proceedings of the 21st International Conference Radioelektronika. Brno (Czech Republic), 2011, p. 211-214. DOI: 10.1109/RADIOELEK.2011.5936469

[19] GUZAN, M. Boundary surface of a ternary memory in the absence of limit cycles, In Proceedings of the 22nd International Conference Radioelektronika 2012. Brno (Czech Republic), 2012, p. 1-4. DOI: 10.1109/RADIOELEK.2011.5936469

[20] GALAJDA, P., ŠPÁNY, V., GUZAN, M. The state space mystery with virtual saddle point in memory cell. In Proceedings of the DSP - MCOM 2005. Košice (Slovakia), 2005, p. 147-150.

[21] GALAJDA, P., GUZAN, M., ŠPÁNY, V. The state space description of the MVL memory circuits. In Proceedings of the International Conference Education, Science and Economics at Universities: Integration to International Educational AREA. Plock (Poland), 2010, p. 351-359. ISBN 978-83-60662-38-0.

[22] TONDL, A. Domains of Attraction for Non-linear Systems. Monographs and memoranda No. 8. National Research Institute for Machine Design, Běchovice, 1970.

[23] PETRŽELA, J. Optimal piecewise-linear approximation of the quadratic chaotic dynamics. Radioengineering, 2012, vol. 21, no. 1, p. 20-28. ISSN 1210-2512.

[24] GALIAS, Z. Basins of attraction for periodic solutions of discretized sliding mode control systems. In Proceedings of IEEE International Symposium on Circuits and Systems (ISCAS). Paris (France), 2010, p. 693-696.

[25] COLOMBO, A., GALVANETTO, U. On the boundaries of basins of attraction in piecewise smooth systems. In Proceedings of the Complexity in Engineering COMPENG '10. Rome (Italy), 2010, p. 100-102. DOI: 10.1109/COMPENG.2010.41

[26] YUJIAO HUANG, ZHANSHAN WANG, HUAGUANG ZHANG, TIE ZHANG. Multistability and enlarged basins of attraction in bidirectional associative memory neural networks. In 
Proceedings of the International Conf. on Networking, Sensing and Control (ICNSC). Chicago (IL, USA), 2010, p. 376-381. DOI: 10.1109/ICNSC.2010.5461531

[27] WENLIAN LU, LILI WANG, TIANPING CHEN. On attracting basins of multiple equilibria of a class of cellular neural networks. IEEE Transactions on Neural Networks, 2011, vol. 22, no. 3, p. 381-394. DOI: $10.1109 / \mathrm{TNN} .2010 .2102048$

[28] RUZ-HERNANDEZ, J. A., SUAREZ-DURAN, M. U, GARCIAHERNANDEZ, R., et al. A comparative analysis-based on basins of attraction for neural associative memories. In Proceedings of the World Automation Congress (WAC). Puerto Vallarta (Mexico), p. 1-6, 2012. ISBN: 978-1-4673-4497-5

[29] KAPITANIAK, T., CZOLCZYNSKI, K. Impacting oscillators the problem of visualization of basins of attraction. In Proceedings of the International Conference Physics and Control. 2003, vol. 2, p. 657-662. DOI: 10.1109/PHYCON.2003.1236912

[30] ŠPÁNY, V., PIVKA, L. Dynamic properties of flip-flop sensors. Journal of Electrical Engineering, 1996, vol. 47, no. 7-8, p. 169178.

[31] KALAKAJ, P., ŠPÁNY, V., ŠOLTYS, R. Flip-flop sensors with feedback. In Proceedings of the International Conference Tesla III Millennium. Belgrade (Yugoslavia), 1996, p. 145-149.

[32] KOLLÁR, M., ŠPÁNY, V., GABAŠ T. Autocompensative system for measurement of the capacitances. Radioengineering, 2002, vol. 11, no. 2, p. 26-30. ISSN 1210-2512.

[33] LEVICKÝ, D., MICHAELI, L., ŠPÁNY, V., PIVKA, L., KALAKAJ, P. Autocompensative system with flip-flop sensor. In Proceedings of the International Conference. Napoli (Italy), 1996, p. $185-189$.

[34] ŠPÁNY, V., PIVKA, L., GALAJDA, P., GUZAN, KALAKAJ, P., ŠAK, A. The calculation of symmetrizing voltage in flip flop sensors. In Proceedings of the International Conference Radioelectronics. Košice (Slovakia), 1994, p. 22-27.

[35] KOLLÁR, M. Flip-Flop sensor controlled by slow-rise control pulse. Radioengineering, 2001, vol. 10, no. 3, p. 34-38. ISSN 1210-2512.

[36] MATSUMOTO, T., CHUA, L., KOMURO, M. The double scroll. IEEE Transactions on Circuits and Systems, 1985, vol.CAS-32, p. 797-818. DOI: $10.1109 /$ TCS.1985.1085791
[37] ŠPÁNY, V., PIVKA, L. Boundary surfaces in sequential circuits. International Journal of Circuit Theory and Applications, 1990, vol. 18 , no. 4, p. 349-360. DOI: 10.1002/cta.4490180404

[38] GALAJDA, P., KOCUR, D. Chua's circuit in spread spectrum communication systems. Radioengineering, 2002, vol. 11, no. 2, p. 6-10. ISSN 1210-2512.

[39] ŠPÁNY, V., GALAJDA, P., GUZAN, M., PIVKA, L., OLEJÁR, M. Chua's singularities: Great miracle in circuit theory. International Journal of Bifurcation and Chaos, 2010, vol. 20, no. 10, p. 2993-3006. DOI: 10.1142/S0218127410027544

[40] PETRŽELA, J., GOTTHANS, T., GUZAN, M. Dynamical tangles in third-order oscillator with single jump function. The Scientific World Journal, 2014, vol. 2014, p. 1-12, DOI: $10.1155 / 2014 / 239407$

[41] GUZAN, M., SOBOTA, B. 3D visualization of Chua's circuit dynamics. Journal of Information, Control and Management Systems, 2010, vol. 8, no. 4, p. 311-316.

[42] KENNEDY, P. Robust op amp realization of Chua's circuit. Frequenz, 1992, vol. 46, no. 3-4, p. 66-80. ISSN: 0016-1136.

[43] PETRŽElA, J., HRUBOŠ, Z., GOTTHANS, T. Modeling deterministic chaos using electronic circuits. Radioengineering, 2011, vol. 20, no. 2, p. 438-444. ISSN 1210-2512.

[44] AZIZ-ALAOUI, M. A. Multispiral chaos. In Proc. of the 2nd Internat. Conf. Control of Oscillations and Chaos. St. Petersburg (Russia), 2000, p. 88-91. DOI: 10.1109/COC.2000.873517

\section{About the Author ...}

Milan GUZAN was born in Snina (Slovak Republic) in 1969. In 1992 he graduated (MSc.) at the Dept. of Radio Electronics, Faculty of Electrical Engineering and Informatics, Technical University of Košice. In 2001 he defended his $\mathrm{PhD}$. in section Electronics. He works as an assistant professor at the Dept. of Theoretical and Industrial Electrical Engineering, FEI TU in Košice. His scientific interests are focused on the analysis of nonlinear circuits and metrology. 